\title{
Travel-Time Difference Extracting in Experimental Study of Rayleigh Wave Acoustoelastic Effect
}

\author{
Hu Eryi and Ying Shao \\ School of Mechatronic Engineering, China University of Mining and Technology, Xuzhou, Jiangsu 221116, China \\ Correspondence should be addressed to Hu Eryi; horyhu@126.com
}

Received 25 January 2014; Accepted 6 March 2014; Published 17 March 2014

Academic Editors: A. Gelfgat and Y.-H. Lin

Copyright ( 2014 H. Eryi and Y. Shao. This is an open access article distributed under the Creative Commons Attribution License, which permits unrestricted use, distribution, and reproduction in any medium, provided the original work is properly cited.

\begin{abstract}
In order to identify the travel-time difference accurately in the experimental study of Rayleigh wave acoustoelastic effect, an experimental system is constructed by the ultrasonic pulser-receiver, digital oscilloscope, Rayleigh wave transmitter and receiver, and a personal computer. And then, the digital correlation method and the Fourier transform frequency analysis method are used to obtain the travel-time difference of the Rayleigh wave corresponding to different subsurface stress conditions. Furthermore, the simulated ultrasonic signals are used to verify the reliability of the two kinds of ultrasonic signal information extracting algorithms, respectively. Finally, the proposed signal processing methods are applied to extract the time delay between different Rayleigh wave signals corresponding to different subsurface stress level.
\end{abstract}

\section{Introduction}

In recent years, stress measurement has been a hot issue in the mechanical engineering. For instance, by using the strain gauge stress measurement is the traditional detection method, which has been widely used in the principal engineering stress estimate. On the other hand, some new nondestructive techniques have been developed to obtain the stress station of the metal or nonmetal material for engineering structure.

After the acoustoelastic effect of the ultrasonic is proposed and proved by the experimental studies $[1,2]$, this type of physical phenomenon is applied to evaluate the stress level of propagation medium. As we know, the longitudinal and transverse ultrasonic waves are used for estimating the average stress of a structure along the thickness direction because of the changing of the propagating characteristic. However, Rayleigh wave, which propagates along the surface of the material, makes it possible to probe the subsurface stress of the detected object.

However, the acoustoelastic effect of longitudinal, transverse ultrasonic wave, and Rayleigh wave is a weak effect caused by the subsurface stress influence. From the previous experimental research, the experimental phenomenon cannot be captured easily. So identifying the difference in time of flight accurately is the key point in the experimental study of acoustoelastic effect. In this paper, two different ultrasonic process methods are discussed to obtain the traveltime difference. The signal process algorithms are verified by digital simulation and experimental approach.

\section{The Acoustoelastic Effect of Rayleigh Wave}

The propagating velocity of the Rayleigh wave in a uniformly deformed solid medium surface is changed due to the subsurface plane stress. Furthermore, this kind of acoustoelastic effect in the steel or aluminium alloy material has been affirmed by many experimental studies [3]. A Rayleigh wave with initial velocity $v$ and stress fields $\sigma$, respectively, would attain a phase shift after propagating in a given distance on the medium surface. According to the discussions about Rayleigh wave acoustoelastic effect conducted by $\mathrm{Hu}$ et al. [4], The variations of the propagation velocity can be expressed as a function of the two nonzero orthogonal subsurface stresses $\sigma_{1}$ and $\sigma_{2}$ as follows:

$$
\frac{\Delta v}{v}=\beta_{1} \sigma_{1}+\beta_{2} \sigma_{2}
$$

where $\beta_{1}, \beta_{2}$ are the acoustoelastic constants which are dependent upon the elastic constants of different propagating medium materials. 
Because of the changing of the Rayleigh wave propagating velocity, there will be a distinct difference in time of flight of the Rayleigh wave propagating along a confirmed distance. From (1), the other expression of the Rayleigh wave acoustoelastic effect is shown as follows:

$$
\Delta t_{1(1,2)}=t_{0} \beta_{1} \sigma_{1}+t_{0} \beta_{2} \sigma_{2},
$$

where $\Delta t_{1(1,2)}$ denotes the difference in time of flight experienced by the Rayleigh wave propagating in the " 1 " direction caused by a biaxial stress applied with principal directions along with " 1 " and " 2 " direction, and $t_{0}$ is the time of flight of the Rayleigh wave which is propagating along the confirmed distance when the object surface is at a zero stress state. In order to get a compact formula, (2) can be briefly written as the following form:

$$
\Delta t_{1(1,2)}=k_{1} \sigma_{1}+k_{2} \sigma_{2} .
$$

$k_{1}$ and $k_{2}$ are two Rayleigh wave acoustoelastic coefficients corresponding to different principal stress directions. Furthermore, the two coefficients are confirmed to different materials in the same engineering conditions. Hence, the two coefficients, $k_{1}$ and $k_{2}$, must be obtained by theoretical deducing or determined by a calibration test. After the difference in time of flight of the Rayleigh wave propagating over a distance along the surface of the medium is obtained, the surface stress can be computed by (3).

However, as the acoustoelastic effect is a weak effect caused by the subsurface stress, the experimental phenomenon cannot be captured easily. From the previous experimental research, it is found that the variation of Rayleigh wave velocity caused by the stress change of $100 \mathrm{MPa}$ is about $0.1 \%$ for aluminium and $0.01 \%$ for steel [5]. In other words, the time difference of the Rayleigh wave propagating is in the range of nanoseconds. So identifying the difference in time of flight accurately is the key point in the experimental study of acoustoelastic effect.

\section{Experimental Set-Up}

The schematic diagram of the Rayleigh wave acoustoelastic effect experimental system is illustrated in Figure 1. Figure 2 is the corresponding physical map of the experimental system. The experimental system is constructed by the ultrasonic pulser-receiver, digital oscilloscope, Rayleigh wave transmitter and receiver, and a personal computer. The ultrasonic pulser-receiver is used to excite the transmitter and receives the signals coming from the receiver. And then, the digital oscilloscope is used for sampling ultrasonic waveforms, and the digitized waveforms are stored in the memory of the computer. In detail, the type of ultrasonic pulser-receiver manufactured by GE PANAMETRICS is MODEL 5800, and the TDS5000B digital oscilloscope is manufactured by TEKTRONIX.

\section{Digital Correlation Method for Measuring the Time Difference}

4.1. Basic Principle. As we know, there is an obvious correlation between the different signals obtained during the same

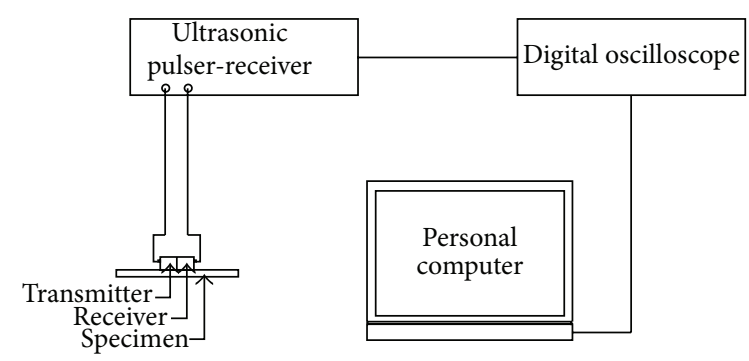

FIGURE 1: Sketch map of the experimental system.

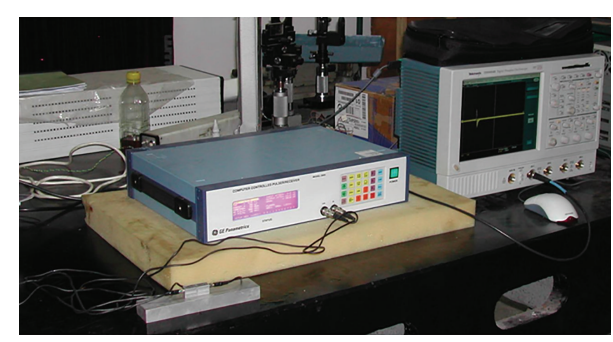

FIgURE 2: Physical map of the experimental system.

experimental procedure. In the traditional signal analysis method, the correlation coefficients can be used to describe the two signal waveforms' similarity. If the signals denoted as $s_{1}(t)$ and $s_{2}(t)$ are assumed as energy limited signals, the correlation coefficient $R_{12}$ can be expressed as follows [6]:

$$
R_{12}=\frac{\int_{-\infty}^{+\infty} s_{1}(t) s_{2}(t) \mathrm{d} t}{\left[\int_{-\infty}^{+\infty} s_{1}^{2}(t) \mathrm{d} t \int_{-\infty}^{+\infty} s_{2}^{2}(t) \mathrm{d} t\right]^{1 / 2}}
$$

Thus, the ultrasonic signals obtained from the stressed surface and the stress-free reference samples have a time delay in the time domain because of the difference of the Rayleigh wave velocity. The difference in time of flight of the Rayleigh wave can be identified by using the previous digital correlation method. The details of the processing procedure of the Rayleigh wave signals are shown as follows. Now, we can mark the ultrasonic signals obtained corresponding to different surface stress situations as $s_{1}(t)$ and $s_{2}(t)$. For instance, $s_{1}(t)$ denotes the signal waveform as the free surface of the detected specimen, and $s_{2}(t)$ denotes the signal waveform as the loaded surface of the detected specimen.

However, by using the proposed experimental system constructed in this thesis, the ultrasonic signal recorded by the digital oscilloscope and computer is a discrete signal. For this kind of discrete signals, the normalized correlation coefficient is written as

$$
\begin{aligned}
R_{12}= & \left(\sum s_{1}(i) s_{2}(i)-\frac{\sum s_{1}(i) \sum s_{2}(i)}{n}\right) \\
& \times\left\{\left[\sum s_{1}^{2}(i)-\frac{\left(\sum s_{1}(i)\right)^{2}}{n}\right]\right.
\end{aligned}
$$




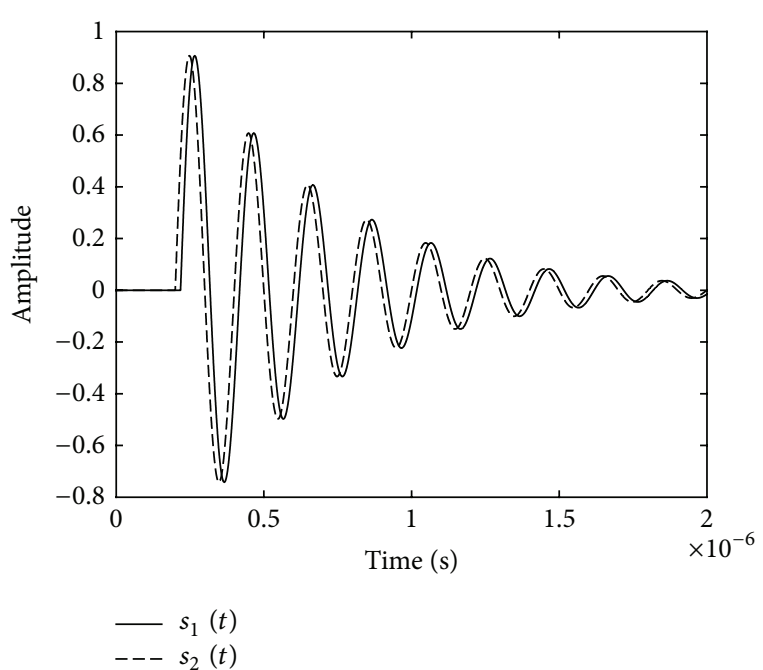

FIGURE 3: The waveform of the two simulated signals.

$$
\begin{array}{r}
\left.\times\left[\sum s_{2}{ }^{2}(i)-\frac{\left(\sum s_{2}(i)\right)^{2}}{n}\right]\right\}^{-1 / 2} \\
(i=1, \ldots, n) .
\end{array}
$$

In the digital signal processing, the sampling period is set as $\delta$, and $n$ is denoted as the correlation calculation window length. By a series of circulatory calculation, if the maximum value of the correlation coefficient is achieved with the discrete data $s_{1}(1) \sim s_{1}(n)$ of $s_{1}(t)$ and $s_{2}(1+k) \sim s_{2}(n+k)$ of $s_{2}(t)$, the corresponding difference in time of flight $\Delta t$ is equal to $k \delta$.

4.2. Simulation Verification of the Algorithm. To verify the reliability of the digital correlation method algorithm, the simulated ultrasonic signals are used to verify the previous proposed algorithm. The sample frequency $f_{s}$ is set as $5 \times$ $10^{9} \mathrm{~Hz}$, and the time delay $\Delta t$ between the two simulated ultrasonic signals is set as $1.8 \times 0^{-8} \mathrm{~s}$. The waveforms of the two simulated signals are shown in Figure 3. The correlation calculation window length is $1.0 \times 10^{-7} \mathrm{~s}$. Finally, the result of the correlation calculation is shown in Figure 4.

From the correlation coefficient calculation result shown in Figure 4, it is obvious that there is a significant peak of the correlation coefficient along the time axis; maximum correlation coefficient corresponding to the time difference $\Delta t$ is $1.8 \times 10^{-8} \mathrm{~s}$. The calculated result is fit well with the theoretical setting value, and the calculation accuracy of the proposed correlation coefficient method is just one sampling period.

4.3. Calculation Method Application for Experimental Results of Ultrasonic Signals. The proposed correlation coefficient method is used to extract the time delay among different ultrasonic signals corresponding to different surface stress level. The detected material is Q235 steel. As shown in Figure 5, a part of intercepted ultrasonic signal is used to

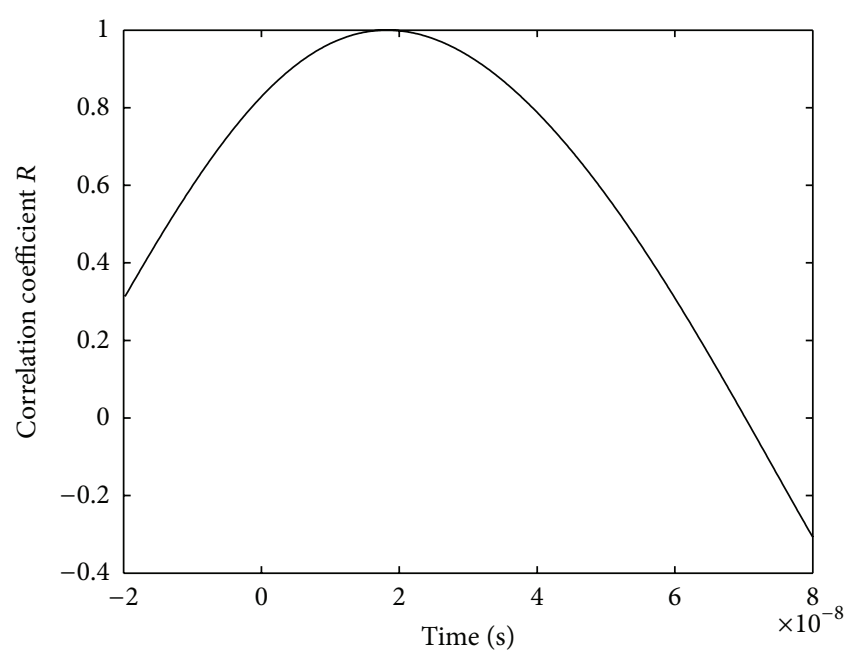

FIgURE 4: The result of the correlation calculation.

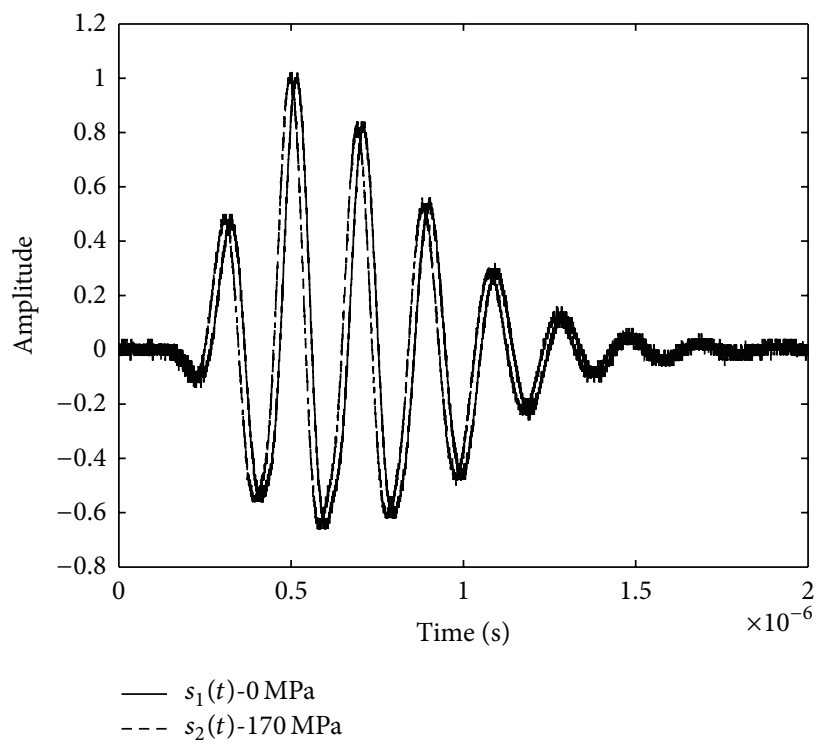

FIGURE 5: A part of intercepted ultrasonic signal.

prove the algorithm, and the sampling frequency is $5 \mathrm{GHz}$. Compared with the simulated signals, high frequency noise is appearing in the actual ultrasonic signal. By using the correlation method to calculate the time difference between two signals, the correlation coefficient distribution is shown in Figure 6.

From the correlation coefficient calculation result shown in Figure 6, there is also a peak value in the correlation coefficient distribution map. The time value corresponding to this peak value is the time difference between the two ultrasonic signals. Thus, we can find that the time delay for this example is $\Delta t=1.78 \times 10^{-8} \mathrm{~s}$.

\section{Frequency Analysis Method for Measuring the Time Difference}

5.1. Basic Principle. Compared with the proposed digital correlation method in the time domain, the time delay 


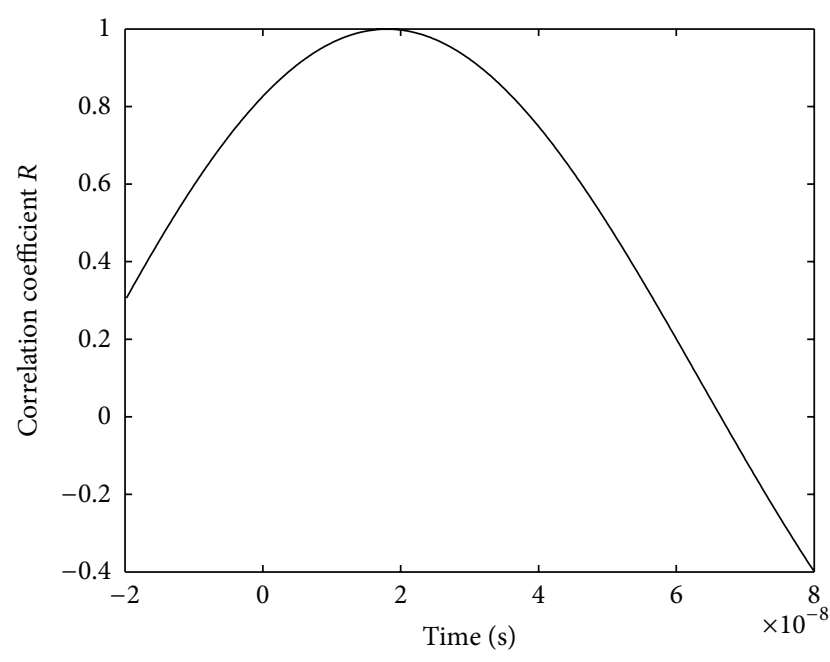

FIgURE 6: The correlation coefficient distribution.

between the different waveforms can also be obtained by the phase extracting method in the frequency domain, such as the Fourier transform technique. As we know, the ultrasonic signals recorded by the experimental system can be approximately expressed as

$$
\begin{aligned}
& s_{1}(t)=a_{1}(t) \cdot \cos \left(2 \pi f_{0} t+\varphi_{1}\right) \\
& s_{2}(t)=a_{2}(t) \cdot \cos \left(2 \pi f_{0} t+\varphi_{2}\right),
\end{aligned}
$$

where $a_{1}(t)$ and $a_{2}(t)$ are the amplitude of the ultrasonic intensity, $f_{0}$ is the principal vibration frequency of the ultrasonic sensors, and $\varphi_{1}$ and $\varphi_{2}$ are the initial phase corresponding to different waveforms. The relationship between the time delay $\Delta t$ and the phase difference $\Delta \varphi$ is shown as follows:

$$
\Delta t=\frac{\varphi_{1}}{2 \pi f_{0}}-\frac{\varphi_{2}}{2 \pi f_{0}}=\frac{\Delta \varphi}{2 \pi f_{0}} .
$$

Hence, we can get the time delay by obtaining the phase difference of the waveforms. Fourier transform method is normally used to extract the phase change $[7,8]$. Equation (6) shows that the signal $\varphi$ is modulated by a constant high-frequency signal $f_{0}$. And then, by using a simple mathematical manipulation, (6) can be written as

$$
\begin{aligned}
& s_{1}(t)=c_{1}(t) \exp \left(j 2 \pi f_{0} t\right)+c_{1}^{*}(t) \exp \left(-j 2 \pi f_{0} t\right), \\
& s_{2}(t)=c_{2}(t) \exp \left(j 2 \pi f_{0} t\right)+c_{2}^{*}(t) \exp \left(-j 2 \pi f_{0} t\right),
\end{aligned}
$$

where $c(t)=[a(t) / 2] \exp [j \varphi]$, and $c^{*}(t)$ is the complex conjugate of $c(t)$. The Fourier transform of $s(t)$ with respect to $t$ becomes

$$
F[s(t)]=C\left(f-f_{0}, t\right)+C^{*}\left(f+f_{0}, t\right),
$$

where $F[]$ and $C()$ represent the Fourier spectra, and $C^{*}$ is the complex conjugate of $C$.

As the frequency of $a(t)$ and $\varphi$ are much lower than $f_{0}$, the function $C\left(f-f_{0}, t\right)$ can be filtered by an adequate window in the frequency domain. And then, $C(f, t)$ can be obtained by spectrum shift center. Taking inverse Fourier transform of $C(f, t)$, we can get $c(t)$. The phase of the waveform is

$$
\begin{aligned}
\varphi(t) & =\arctan \left\{\frac{\operatorname{Im}[c(t)]}{\operatorname{Re}[c(t)]}\right\}+2 n \pi \\
& =\arctan \frac{G}{F}+2 n \pi=\varphi^{\prime}(t)+2 n \pi,
\end{aligned}
$$

where $F=\operatorname{Re}[c(t)]$ and $G=\operatorname{Im}[c(t)]$ represent the real and imaginary parts of $c(t)$, respectively. $\varphi^{\prime}(t)$ is the principal phase which is in the range of $[-\pi, \pi]$. A phase unwrapping procedure $[9,10]$ should be taken to get the continuous actual phase distribution $\varphi(t)$.

By using the previous phase extracting proceeding, the phase values corresponding to two different ultrasonic waveforms can be extracted, and then the time delay $\Delta t$ between the waveforms is obtained by (7).

5.2. Simulation Verification of the Algorithm. In this section, the digital simulation method is also applied to verify the proposed phase extracting algorithm. In order to compare the two different time difference algorithms in this thesis, the simulated signals' sample frequency $f_{s}$ is also set as $5 \times$ $10^{9} \mathrm{~Hz}$, and the time delay $\tau$ between the two simulated ultrasonic signals is set as $1.8 \times 10^{-8} \mathrm{~s}$, too. The waveforms of the two simulated signals have been given as shown in Figure 3. The procedures and results of the simulated calculation are shown in Figures 7 11. For instance, Figures 7 and 9 are the extracted original phase distribution of the simulated signals corresponding to $s_{1}(t)$ and $s_{2}(t)$. And then, the traditional phase unwrapping algorithm [7] is used to obtain the unwrapped phase distributions, which are shown in Figures 8 and 10. Figure 11 is the phase difference between $s_{1}(t)$ and $s_{2}(t)$, from which it is obvious that the average value of the phase difference is about $\Delta \varphi=0.5484$. Finally, by submitting the phase value into (7) the time delay $\Delta t$ between the waveforms is about $1.75 \times 10^{-8}$. Compared with the original time delay value, it is found that there is a calculation error, and the relative error is about $2.78 \%$. However, this error level can be accepted in the practical engineering detection.

5.3. Calculation Method Application for Experimental Results of Ultrasonic Signals. The proposed phase extracting method is used to extract the time delay among different ultrasonic signals corresponding to different surface stress level. The ultrasonic signals are also shown in Figure 5, and the sampling frequency is $5 \mathrm{GHz}$. Furthermore, the high frequency noise in the actual ultrasonic signal can be reduced among the proceeding of the Fourier transform. The proposed method is applied to calculate the time difference between two signals, and the calculation procedure is as same as the previous simulation calculation. The experimental results are shown in Figures 12, 13, 14, 15, and 16. The average value of the phase difference between $s_{1}(t)$ and $s_{2}(t)$ shown in Figure 16 is $\Delta \varphi=0.5620$. Following (7), the time delay $\Delta t$ between the waveforms is $1.79 \times 10^{-8}$. 


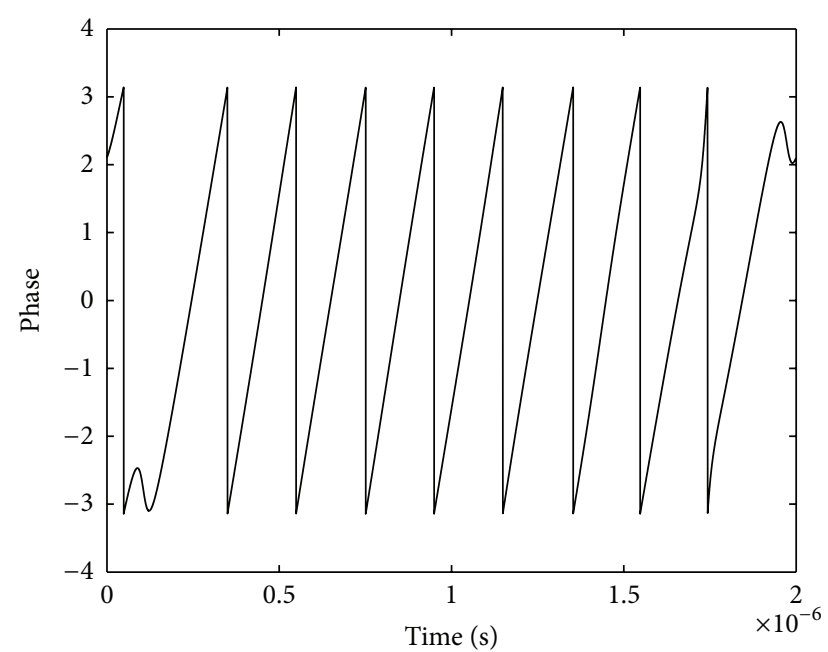

FIGURE 7: Original phase distribution of simulated signal $s_{1}(t)$.

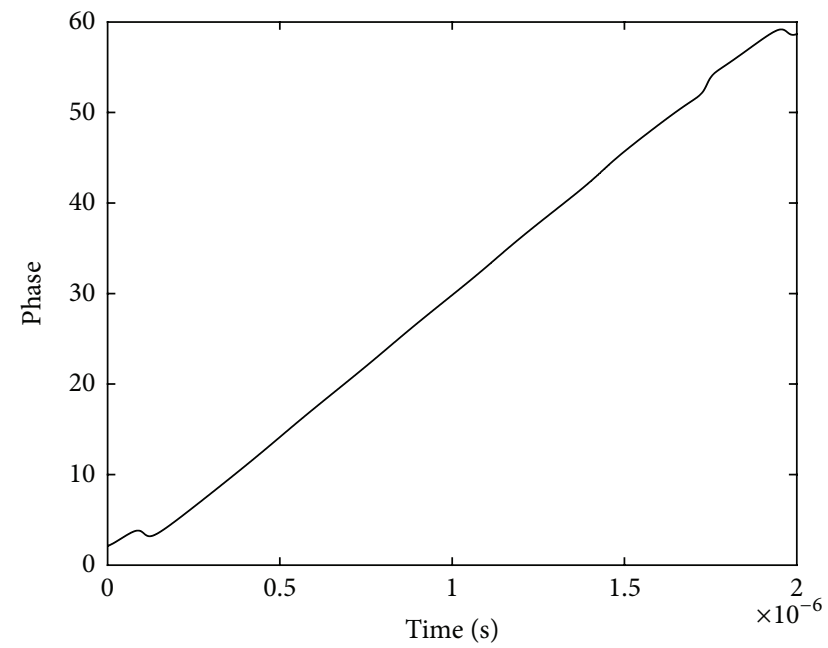

FIGURE 8: Unwrapped phase distribution of simulated signal $s_{1}(t)$.

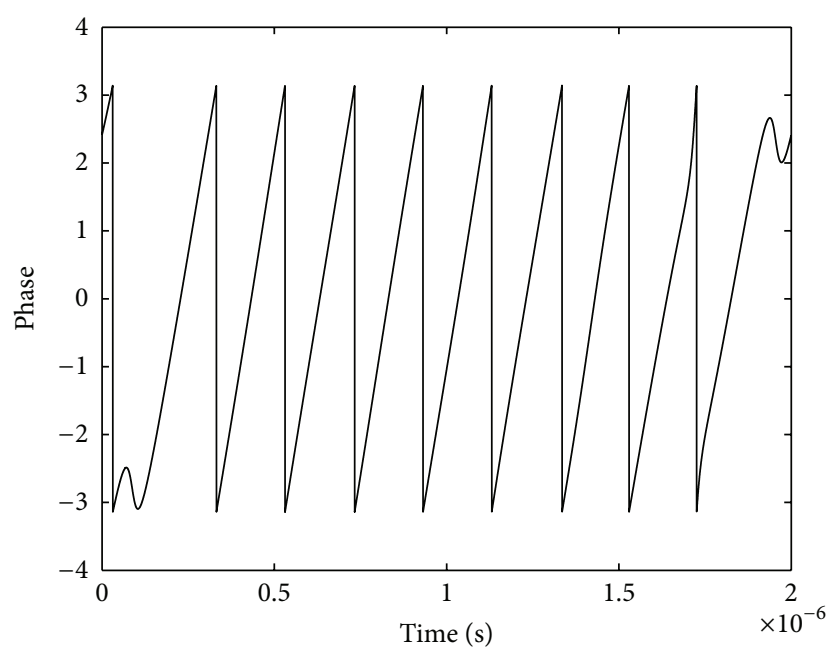

FIGURE 9: Original phase distribution of simulated signal $s_{2}(t)$.

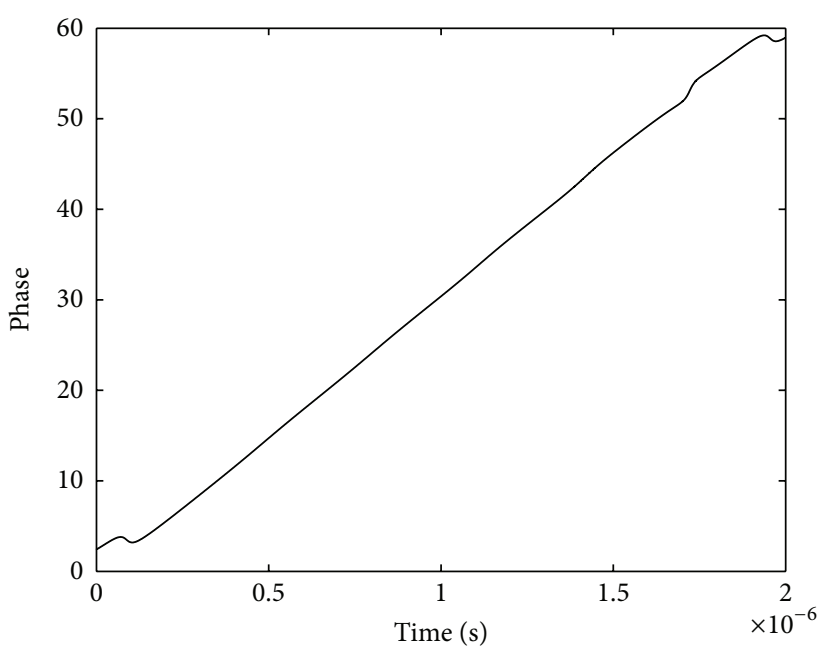

FIGURE 10: Unwrapped phase distribution of simulated signal $s_{2}(t)$.

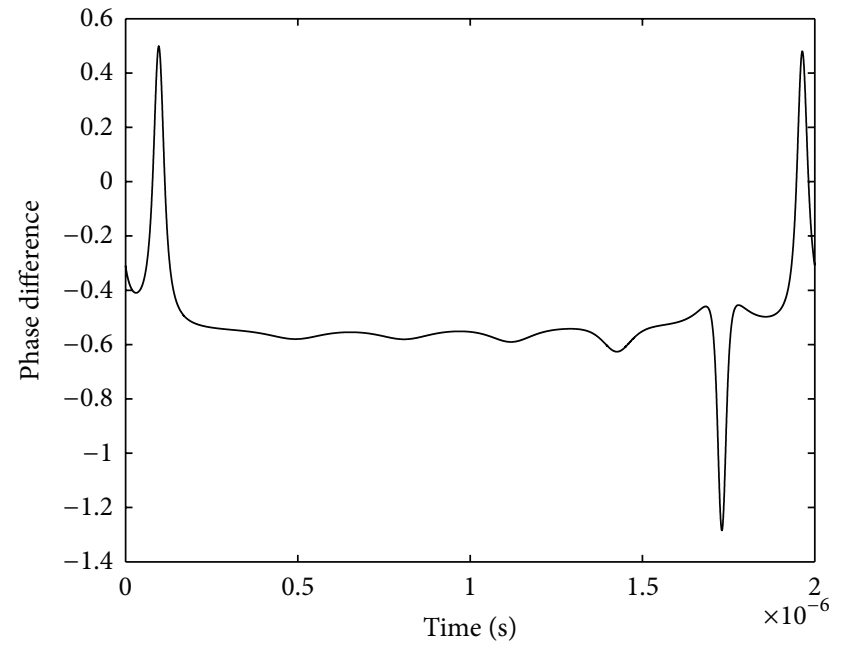

FIGURE 11: Phase difference between simulated signals $s_{1}(t)$ and $s_{2}(t)$

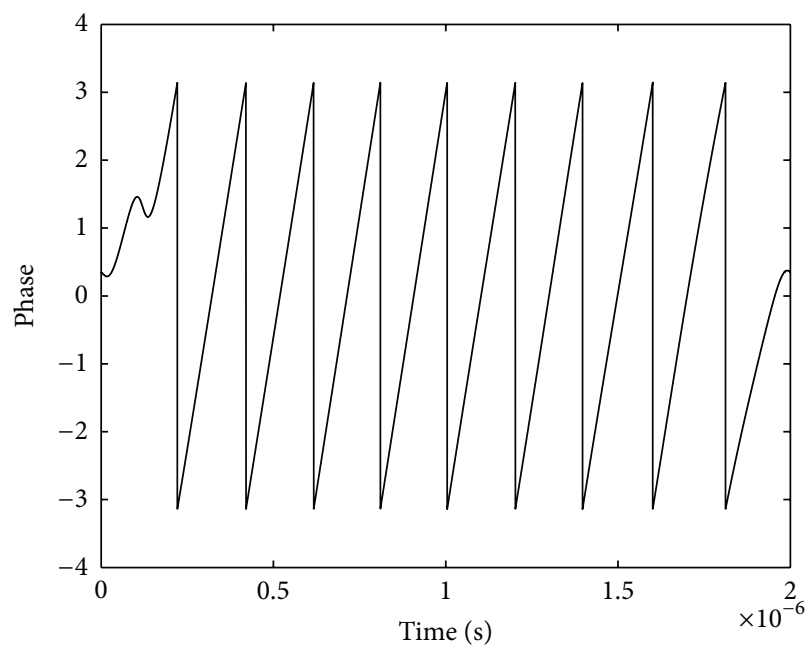

FIGURE 12: Original phase distribution of ultrasonic signal $s_{1}(t)$. 


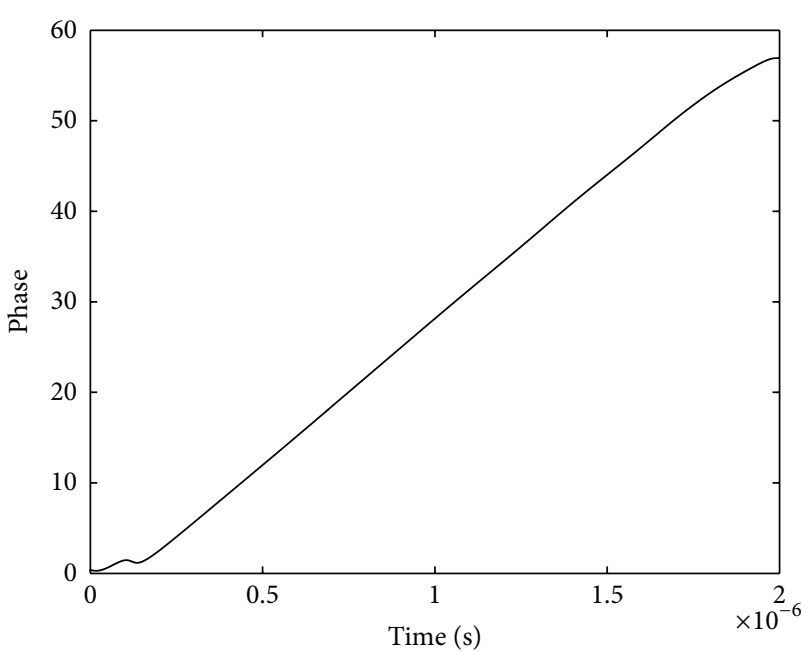

FIGURE 13: Unwrapped phase distribution of ultrasonic signal $s_{1}(t)$.

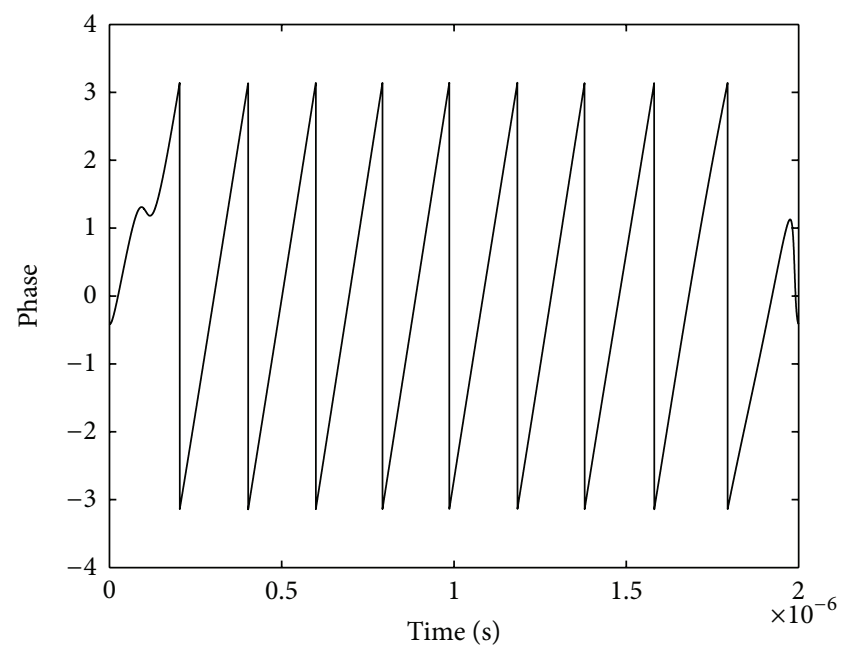

FIGURE 14: Original phase distribution of ultrasonic signal $s_{2}(t)$.

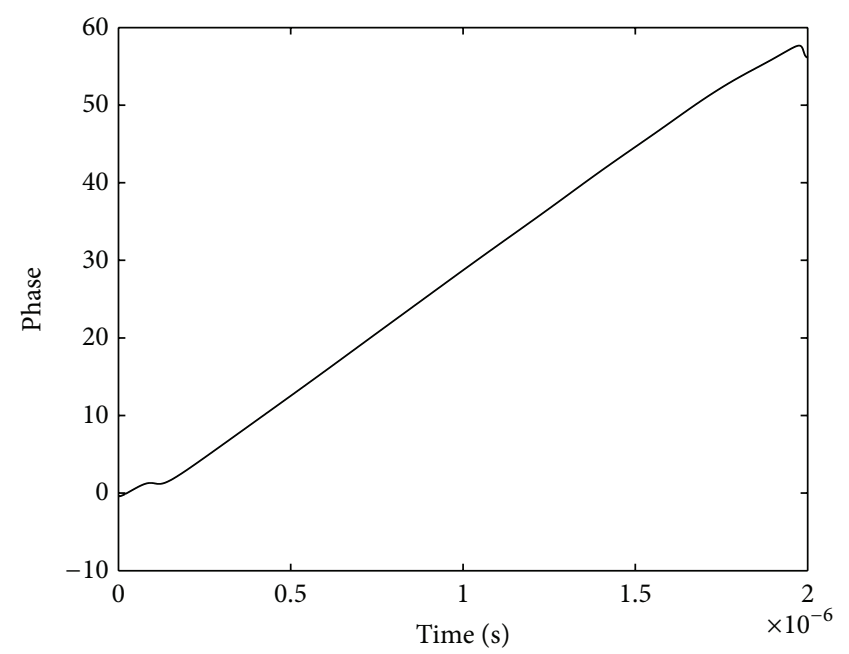

FIGURE 15: Unwrapped phase distribution of ultrasonic signal $s_{2}(t)$.

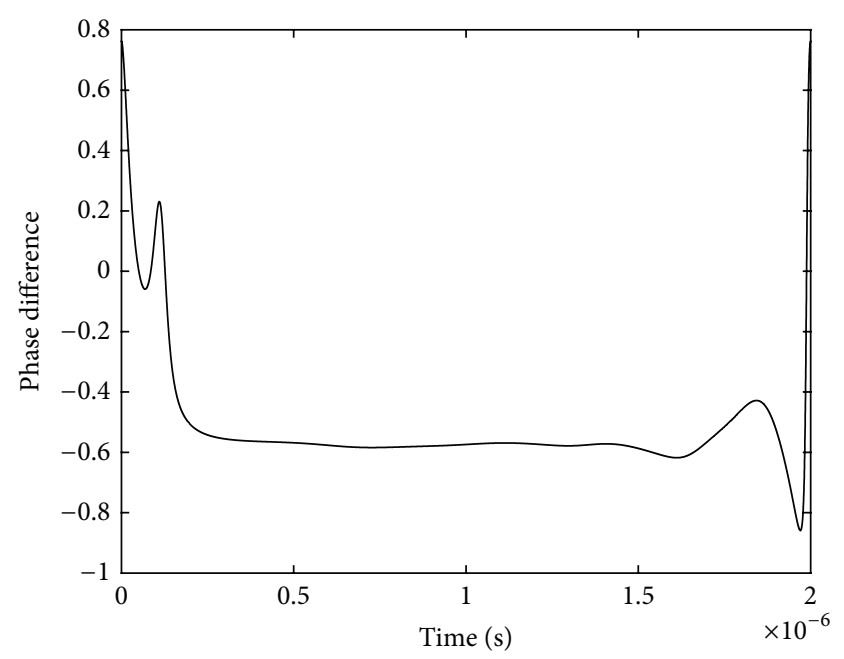

Figure 16: Phase difference between ultrasonic signals $s_{1}(t)$ and $s_{2}(t)$.

\section{Conclusion}

Through the simulation and experimental analysis, the following conclusions can be drawn.

(1) For the digital correlation method, it is obvious that there are significant peaks of the correlation coefficient along the time axis in the correlation calculation result maps. In the simulation analysis, the calculated result is fit well with the theoretical setting value, and the calculation accuracy of the proposed correlation coefficient method is just one sampling period. In the experimental application of Q235 steel, the traveltime difference value corresponding to $0 \mathrm{MPa}$ and $170 \mathrm{MPa}$ is $\Delta t=1.78 \times 10^{-8} \mathrm{~s}$.

(2) For the Fourier transform frequency analysis method, after comparing the calculation value with the original time delay value, it is found that there is a calculation error, and the relative error is about $2.78 \%$. However, this error level can be accepted in the practical engineering detection. In the experimental application of Q235 steel, the average value of the phase difference between $s_{1}(t)$ and $s_{2}(t)$ corresponding to $0 \mathrm{MPa}$ and $170 \mathrm{MPa}$ is $\Delta \varphi=0.5620$. The time delay $\Delta t$ between the waveforms is $1.79 \times 10^{-8}$.

\section{Conflict of Interests}

The authors declare that there is no conflict of interests regarding the publication of this paper.

\section{Acknowledgments}

The authors gratefully acknowledge support from National Natural Science Foundation of China under Grant no. 51205396, Science Foundation of Jiangsu Province under Grant no. BK2012130, Fundamental Research Funds for the Central Universities under Grant no. 2012QNA20, and a 
project funded by the Priority Academic Program Development of Jiangsu Higher Education Institutions.

\section{References}

[1] M. Hirao, H. Fukuoka, and K. Hori, "Acoustoelastic effect of Rayleigh surface wave in isotropic material," Journal of Applied Mechanics, Transactions ASME, vol. 48, no. 1, pp. 119-124, 1981.

[2] J. J. Ditri, "Determination of nonuniform stresses in an isotropic elastic half space from measurements of the dispersion of surface waves," Journal of the Mechanics and Physics of Solids, vol. 45, no. 1, pp. 51-66, 1997.

[3] D. Husson, "A perturbation theory for the acoustoelastic effect of surface waves," Journal of Applied Physics, vol. 57, no. 5, pp. 1562-1568, 1985.

[4] E. Hu, Y. He, and Y. Chen, "Experimental study on the surface stress measurement with Rayleigh wave detection technique," Applied Acoustics, vol. 70, no. 2, pp. 356-360, 2009.

[5] A. Zeiger and K. Jassby, "Measurement of acoustoelastic coefficients of Rayleigh waves in steel alloys," Journal of Nondestructive Evaluation, vol. 3, no. 2, pp. 115-124, 1982.

[6] H. Yuming, "Digital shearing speckle correlation fringes pattern formed by using linear correlation calculating method," Acta Photonica Sinica, vol. 24, no. 1, pp. 13-17, 1995.

[7] E. Hu and Y. He, "Surface profile measurement of moving objects by using an improved $\pi$ phase-shifting Fourier transform profilometry," Optics and Lasers in Engineering, vol. 47, no. 1, pp. 57-61, 2009.

[8] D. W. Robinson and G. T. Reid, Interferogram Analysis: Fringe Pattern Measurement Techniques, Institute of Physics, Bristol, UK, 1993.

[9] X. Su, W. Chen, Q. Zhang, and Y. Chao, "Dynamic 3-D shape measurement method based on FTP," Optics and Lasers in Engineering, vol. 36, no. 1, pp. 49-64, 2001.

[10] H.-M. Yue, X.-Y. Su, and Y.-Z. Liu, "Fourier transform profilometry based on composite structured light pattern," Optics and Laser Technology, vol. 39, no. 6, pp. 1170-1175, 2007. 

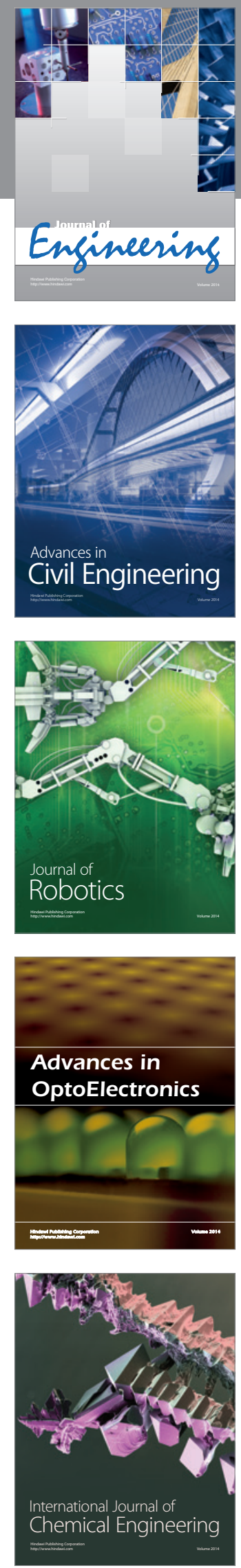

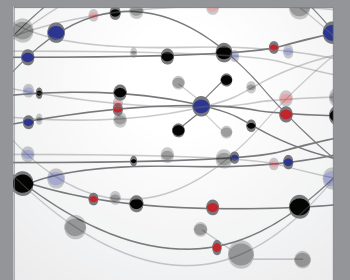

The Scientific World Journal
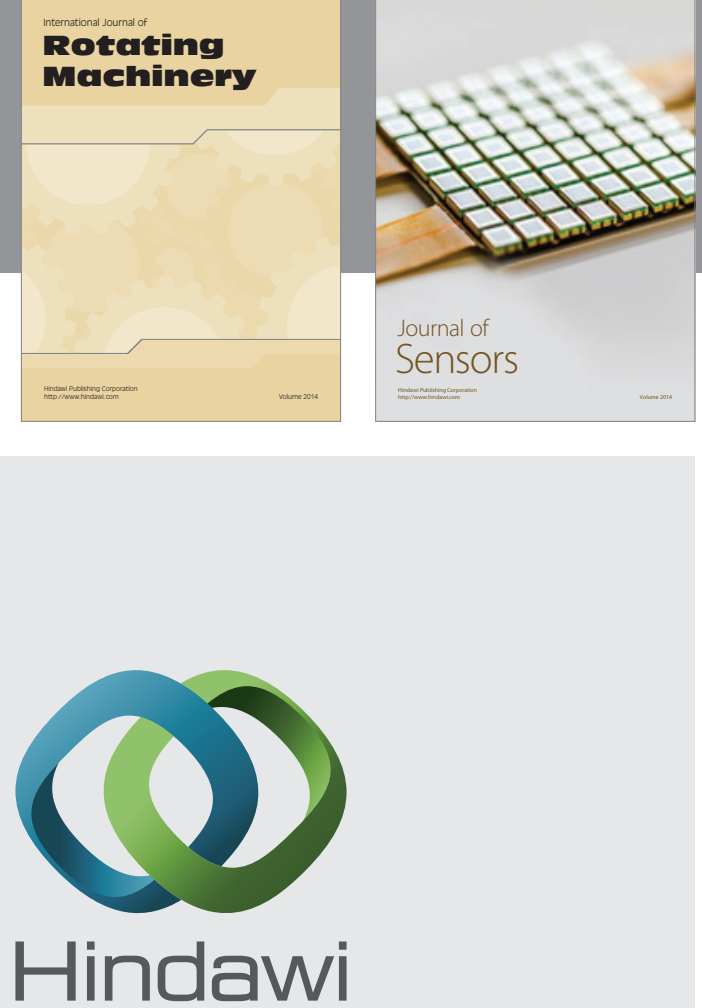

Submit your manuscripts at http://www.hindawi.com
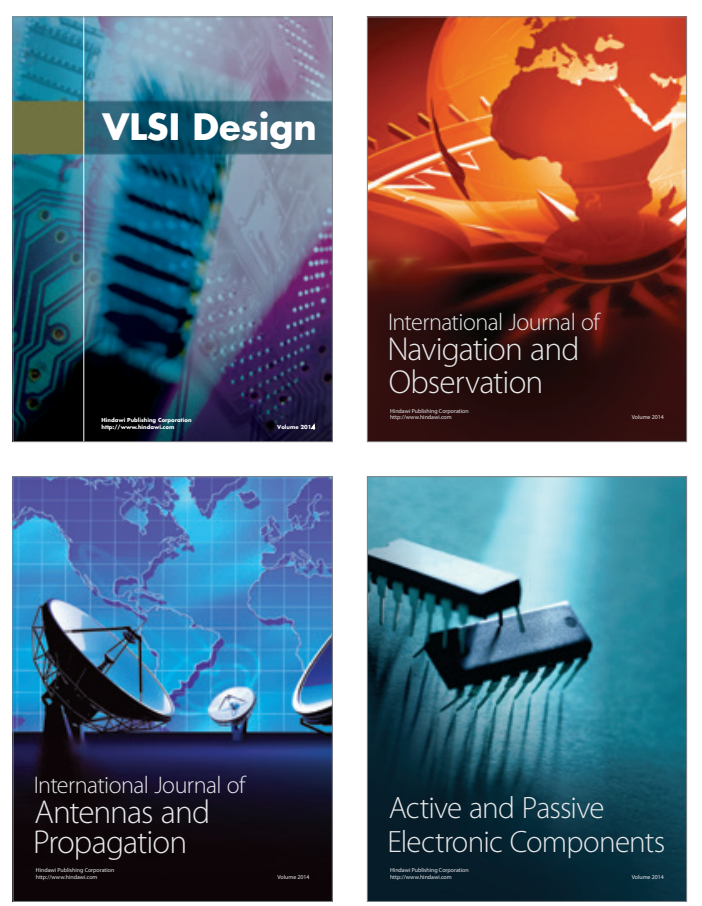
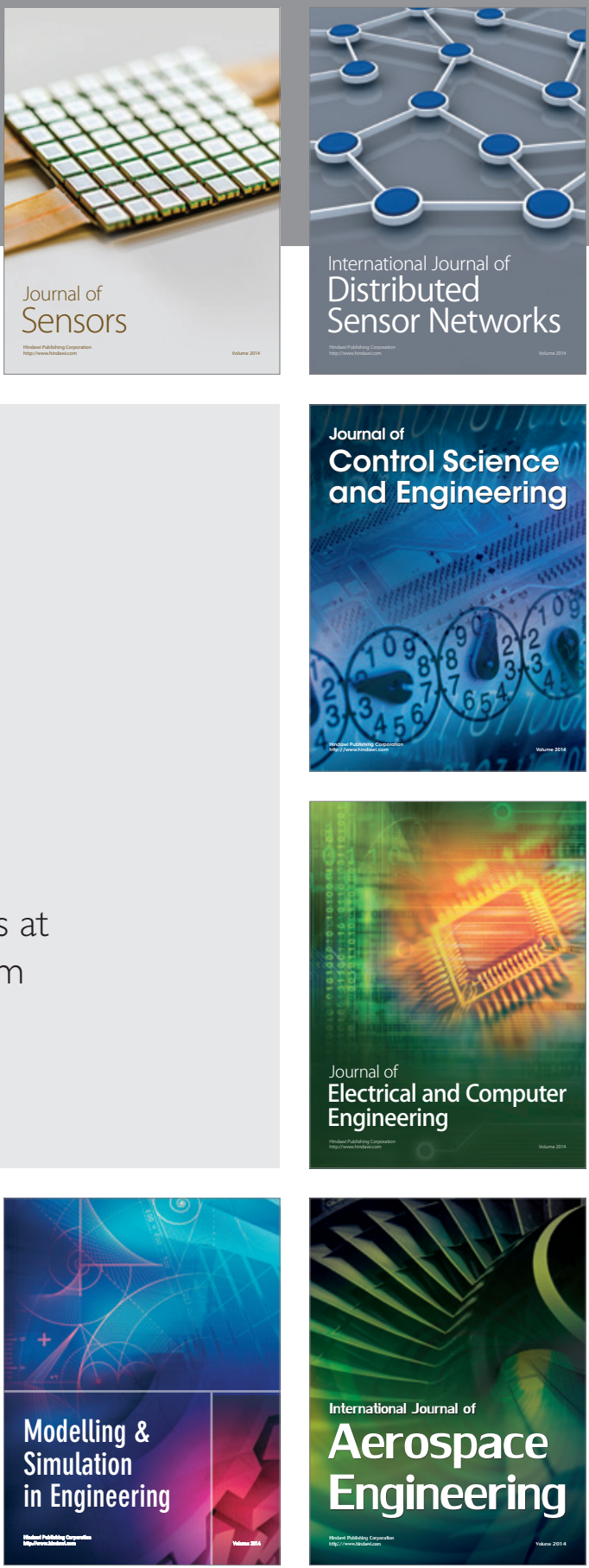

Journal of

Control Science

and Engineering
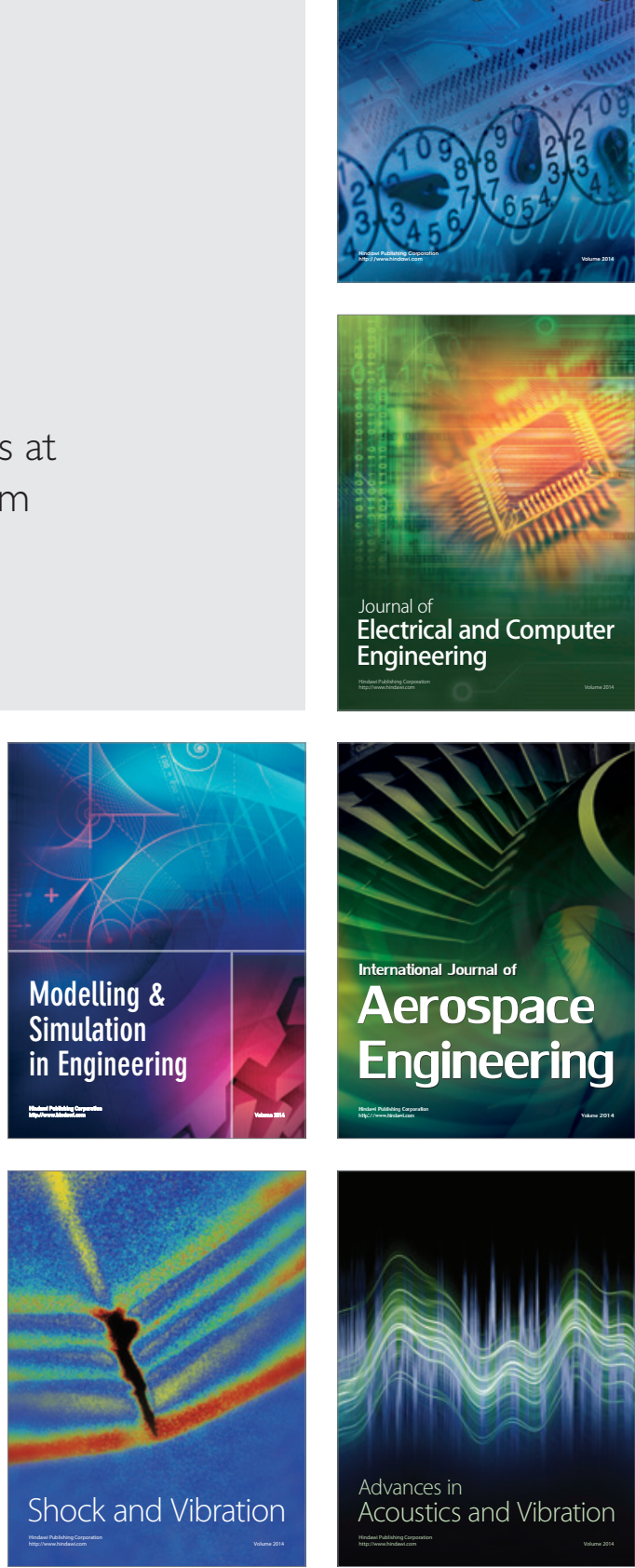